\title{
Transgenerational epigenetic effects on animal behaviour
}

\author{
Per Jensen
}

\section{Linköping University Post Print}

\section{Tweet}

N.B.: When citing this work, cite the original article.

Original Publication:

Per Jensen , Transgenerational epigenetic effects on animal behaviour, 2013, Progress in Biophysics and Molecular Biology, (113), 3, 447-454.

http://dx.doi.org/10.1016/j.pbiomolbio.2013.01.001

Copyright: Elsevier

http://www.elsevier.com/

Postprint available at: Linköping University Electronic Press

http://urn.kb.se/resolve?urn=urn:nbn:se:liu:diva-102253 
Transgenerational epigenetic effects on animal behaviour

\section{Per Jensen}

IFM Biology, AVIAN Behaviour Genomics and Physiology group

Linköping University

58183 Linköping

Sweden

Email: perje@ifm.liu.se 


\section{Abstract}

Over the last decade a shift in paradigm has occurred with respect to the interaction between environment and genes. It is now clear that animal genomes are regulated to a large extent as a result of input from environmental events and experiences, which cause short- and long-term modifications in epigenetic markings of DNA and histones. In this review, the evidence that such epigenetic modifications can affect the behaviour of animals is explored, and whether such acquired behaviour alterations can transfer across generation borders. First, the mechanisms by which experiences cause epigenetic modifications are examined. This includes, for example, methylation of cytosine in CpG-positions and acetylation of histones, and studies showing that this can be modified by early experiences. Secondly, the evidence that specific modifications in the epigenome can be the cause of behaviour variation is reviewed. Thirdly, the extent to which this phenotypically active epigenetic variants can be inherited either through the germline or through reoccurring environmental conditions is examined. A particularly interesting observation is that epigenetic modifications are often linked to stress, and may possibly be mediated by steroid effects. Finally, the idea that transgenerationally stable epigenetic variants may serve as substrates for natural selection is explored, and it is speculated that they may even predispose for directed, non-random mutations. 


\section{Introduction}

Ever since the advent of evolutionary theory and its merging with genetics, it has been a widely accepted truth that information can only flow in the direction from DNA to phenotype and never the opposite way. In other words, no traits acquired during life as a result of environmental events are able to affect the structure or function of the DNA. Hence, according to this established view, acquired variations in phenotype, such as behaviour, cannot be transmitted to the next generation. For example, Futuyma in his seminal 1997 textbook on Evolutionary Biology dismissed the possibility of such transmission vividly: "Evidence for quasi-Lamarckian phenomena is still occasionally reported (...), but no experiments have yet conclusively demonstrated that the environment can act through the soma to direct hereditary changes in an adaptive fashion" (Futuyma, 1997). It is noteworthy that Futuyma mentions epigenetics in only a few sentences en passant in this almost 800 page volume; in his defence, one should of course recognize that most of the work on transgenerational effects on behaviour was done after its publication.

The citation and its comparatively recent age demonstrate the rapid shift in paradigm that biology is facing in the $21^{\text {st }}$ century. Only fifteen years after the quotation above, few in the field would fully subscribe to the view of Futuyma or question the fact that acquired traits will sometimes be mirrored in later generations. Rather, current debate concerns matters of definition and distinction: when considering transgenerational effects, should one limit this to true transmission via the germline or should we also include modifications recurring in each generation as a consequence of, for example, parental care? How many generations do we need to consider in order to have demonstrated a true germline mediated inheritance? How should cultural phenomena be dealt with in this context? And how should we distinguish and account for all pathways in the three-way genotype-epigenotype-environment interaction?

This review will focus on the transmission of behavioural variation across generations in animals. Some of this variation will depend on genetic polymorphisms in DNA-sequence, others on epigenetic variation, which may 
sometimes be transferred via the germ-line. Some of the variation can be maintained across generational borders by means of parental effects or other cultural phenomena. One might further speculate on the possibilities that cultural transmission may predispose for epigenetic modifications (and eventually for genetic mutations), a subject which will be briefly touched upon.

\section{What is behaviour?}

Behaviour is indeed a fuzzy phenotype. The wide range of phenomena, which we refer to by the term include things as disparate in function and organisational level as eye blinking, scratching and preening, mate selection and mating, nest building, migration and social organisation. Barnard, in his textbook on animal behaviour, quotes a definition of behaviour offered by the psychologists Skinner and Hebb: "all observable processes by which an animal responds to perceived changes in the internal state of its body or in the external world" (Barnard, 2004). It goes without saying that great care is needed in defining which aspect and level of behaviour one considers in a particular experiment.

Some behaviour is carried out instinctively by an animal, in the sense that no prior learning is necessary to allow its full expression, whereas other behaviour is only shown by individuals with a particular type of experience what we often refer to as learnt behaviour. However, it is a well known fact in behavioural sciences that the ability to learn, as well as the timing and consistency of learning, is heavily dependent on the genotype of the individual (Pearce, 2008). Hence, all behaviour variation depends on genetic variation at least to some extent (Jensen, 2006).

Given this intense interaction of genes and environment, it is not surprising that behaviour varies extensively even within very narrowly defined cohorts of animals - even same-species animals of the same age and sex will differ in their responses to standardised stimuli. The sometimes ungrateful task of the behaviour geneticist is to tease apart the role of genetic and environmental variation in this, and eventually to locate causal genes and understand the mechanisms of specific polymorphisms in causing the observed variation. And 
with the growing awareness of the importance of epigenetic variation, we of course want to find out more about how this affects behaviour as well.

\section{Epigenetics - orchestration of the genome}

As discussed in the extensive review by Jablonka and Raz, the concept "epigenetics" has gradually evolved towards a more mechanistically defined one than it was originally given when coined by Waddington in the 1940-ies (Jablonka, 2009). Broadly, it now generally refers to all the mechanisms affecting how DNA is expressed and translated. Most interest has been given to two phenomena: methylation of cytosines in $\mathrm{CpG}$ positions, and different chemical modifications of histones, such as methylation and acetylation (Richards, 2006).

CpG-dinucleotides often occur in repeated sequences, referred to as $\mathrm{CpG}$ islands, and these are ubiquitous in promoter regions. When cytosines in this position are methylated, it often causes down-regulation, or even silencing, of the gene (Richards, 2006). In a similar way, histone modifications may cause reduction of transcriptional activity, and as Richards explains, this is often coupled and steered by cytosine methylation. Furthermore, the control and mediation of sites for epigenetic modification appears to be carried out partly by small interfering RNAs, which therefore could also be considered important epigenetic players (Richards, 2006).

The pattern of epigenetic marks - the epigenome - can be thought of as the orchestration of the genome, hence governing the timing and extent of gene expression patterns. It is well established that behavioural variation often correlates with variation in gene expression (Hofmann, 2003; Jöngren, Westander, Nätt, \& Jensen, 2010), even if the mechanisms are so far not well understood. Therefore, epigenetic mechanisms should potentially be able to affect phenotypes such as behaviour by causing alterations in the expression of relevant genes or gene clusters (Jablonka, 2009). A relatively recent review paper, based on a symposium on behaviour epigenetics, claimed to have been able to find only a total of 96 papers dealing with this topic (Lester et al., 2011). This small number reflects the novelty of the area, but in all fairness, the criteria 
for inclusion in the list depend to a large extent on the definition of "epigenetics", and there are actually quite a few more articles dealing with transgenerational effects and behavioural effects of differential gene expression (and perhaps a few in the list which do not relate to behaviour as defined here).

Epigenetic marks can be thought of mainly as three different classes, which Richards has termed obligatory, facilitated and pure (Richards, 2006). The obligatory ones are strictly determined by genotype, either in cis or trans, and hence a particular locus in a specific cell type will always carry a typical epigenetic signature. It has been found that more than $80 \%$ of the genome-wide variation in methylation patterns can be explained by differences in genotype in humans (Gertz et al., 2011). In accordance with this, we found that several genes showing differential methylation between brains of domesticated chickens and their ancestor, the Red Junglefowl, maintained their methylation differences over eight generations of intercrossing, and some of them behaved as true epialleles, with a mendelian inheritance pattern, which strongly indicates that they were under genetic control (Nätt et al., 2012).

The pure marks are essentially independent of genotypes outside the affected locus, while facilitated epigenotypes depend in a probabilistic manner on genotype. From the perspective of epigenetic effects on behaviour and possible transgenerational effects, we are of course most interested in the facilitated and pure epigenetic variants, since these are most likely to be dynamic in response to external events.

The question of whether and how epigenetics affects behaviour and to what extent it can cause transgenerational effects can be split into a number of sub-questions: What is the evidence that ontogenetic experiences can modify epigenetic states? Can specific epigenetic variations be linked to specific behavioural differences? And, can epigenetic modifications be inherited, causing transgenerational effects? In the following, each of these questions will be addressed. For a reliable demonstration of epigenetic, transgenerational effects on behaviour, we need evidence for all three processes: A specific experience should be related to a particular epigenetic modification; specific epigenetic 
marks should be related to predictable behavioural effects; and epigenetic marks acquired through experience and affecting behaviour should be detectable in later generations. This is broadly outlined in fig 1.

\section{How is the epigenome affected by experiences?}

The first, and crucial issue in the context of transgenerational effects is which evidence we have that events during an individual's lifetime can modify its epigenetic profile for a sustained period of time. Much of the data in support of such an effect, particularly in rodents, have been extensively summarised in a couple of recent reviews (Curley, Jensen, Mashoodh, \& Champagne, 2011; Kappeler, 2010). It is of course obvious that many pertinent stimuli, for example associated with stress, aggression or reproduction, will cause transient changes in gene expression profiles in relevant brain regions, probably mediated by a range of mechanisms, allowing proper physiological and behavioural responses. Some of these mechanisms would clearly qualify as epigenetic. But can different experiences lead to long-time, persistent epigenetic changes, perhaps allowing life-long modifications in stress response patterns?

One of the more compelling series of experiments showing epigenetic modifications as a result of specific experiences is the one by Meaney and coworkers, investigating the long-term consequences of different types of early maternal care in rats. Rat mothers were initially found to exert different levels of care (Meaney, 2001). Whereas some performed a lot of nursing and grooming of the pups, others were less considerate. Meaney and his collaborators found that pups raised by more careful mothers showed an increased resilience to later stressful events, and that this was mediated by a modified expression pattern in genes involved in the stress response system, as well as in genes governing hippocampal synaptic development.

In later experiments, the mechanisms have been explored at depth. Weaver and co-workers split rat mothers into those showing high levels of grooming and arched-back nursing ("high-LG-ABN") and those showing low levels ("low-LG-ABN") (Weaver et al., 2004). Looking in the hippocampus, they 
found distinctive differences in DNA-methylation between the offspring of each class of mothers, and this was associated with alterations in histone acetylation and binding of the transcription factor NGFI-A (nerve growth factor-inducible protein A; also known as egr-1, krox-24, zenk, and zif-268) to the glucocorticoid receptor-gene (Nr3c1). The effect was limited to experiences during the first weeks of life, which coincides with the timing when the maternal behaviour shows most individual variation.

The epigenetic differences persisted into adulthood, and could be reversed by cross-fostering (as well as by pharmacological inhibition of histone acetylation), which provides compelling evidence that it is the actual experiences of different maternal quality which cause the effects. Corticosterone response to restraint stress was smaller in adult offspring of "high-LG-ABN" mothers, showing long-lasting effects of the epigenetic modifications. Furthermore, the mode of action was revealed in a series of experiments showing that the inhibition of NGFI-A binding to its consensus sequence by DNA methylation causes the cascade of events eventually leading to the phenotypic effects (Weaver et al., 2007).

The same group went on to look at the entire hippocampal transcriptome in adult rats and found more than 900 genes which were differentially expressed according to level and quality of the early maternal care they had experienced (Weaver, Meaney, \& Szyf, 2006). Inhibition of histone deacetylation and central infusion of a methyl donor reversed the effects of early care on adult stress reactions in an open field, again demonstrating the long-lasting epigenetic effects of post-natal experience.

Not only stress related genes can be epigenetically modified by early experience. Champagne and co-workers studied the programming of the estrogen receptor ER $\alpha$ by maternal care in rats, and whether its differential expression is related to differences in the methylation of its promoter (Champagne et al., 2006). Cross-fostering experiments showed a clear relationship between maternal care and expression levels of $E R \alpha$ in the medial preoptic area (MPOA) of the brain, where offspring fostered by "low-LG-ABN" 
mothers had a lower expression. This was apparently due to increased methylation of the $E R \alpha 1 b$ promoter in these rats. Similarly, Roth, Lubin, , Funk \& Sweatt, (2009) found that early experiences can cause long term epigenetic modifications of $B D N F$, a gene involved in many different neural processes.

Some studies indicate that the shaping of the epigenome starts very early, even before birth. In one study, mice were given alcohol during the first part of gestation, and the effect on offspring methylation was assessed by the expression of the epigenetically regulated allele Agouti Viable Yellow (Kaminen-Ahola et al., 2010). Prenatal alcohol exposure caused hypermethylation and a substantial transcriptional silencing of the allele, leading to a higher number of mice with agouti-coloured fur. The authors suggest that the alcohol exposure may have had a broad effect on epigenetic marks, consistent with postnatal growth restriction and craniofacial abnormalities also found in the offspring.

In humans, observations have been done which strikingly resemble the earlier mentioned effects of mothering in rats. Mothers with a more pronounced depressive or anxious mood during the last third of pregnancy had babies, which at three months of age showed an increased cortisol stress response (Oberlander et al., 2008). Although it can not be excluded that this was caused by a strict genetic effect, it is interesting that the mood differences correlated with hypermethylation of the human glucocorticoid receptor gene $N R 3 C 1$, at a predicted NGFI-A binding site.

In line with this epigenetic link between early experiences and later stress responses in humans, McGowan and coworkers examined hippocampal samples from suicide victims which had a history of childhood abuse, and found decreased levels of glucocorticoid receptor mRNA and also increased methylation of an NR3C1 promoter (McGowan et al., 2009). Patch-methylated contructs indicated that abused persons had a very similar downregulation of the NGFI-A binding as found in children of depressed mothers and offspring of less careful rat mothers. Again, a direct genetic effect can not be excluded, but the findings remain highly suggestive. 
Altogether, there is extensive suggestive evidence that experiences during different life periods, including before birth, can cause lasting modifications in epigenetic marks. The next question is the extent to which such epigenetic variations are related to specific phenotypic traits and behaviour.

\section{Does epigenetic variation affect behaviour?}

As was obvious from the previous section, acquired epigenetic variants are often linked to phenotypic variation. The effects of early mothering style in rats not only changes the epigenome, it is also related to various changes in stress responses and in maternal behaviour in the offspring (Champagne et al., 2006; Oberlander et al., 2008; Weaver et al., 2004). Francis and co-workers crossfostered mouse embryos from different selection lines already at implantation, and found that the behaviour of these animals resembled the mothers which had fostered them more than their genetic sisters and brothers (Francis, Szegda, \& Campbell, 2003). Although the authors did not specifically examine the mechanisms involved, it is highly likely that epigenetic factors played a crucial role in this aspect.

Hence, induced epigenetic variation is probably a potent mediator of behavioural differences. In the present section, I will examine the further evidence that epigenetic variation is linked - or even causal - to specific behaviours.

There is ample data supporting epigenetic causes of different psychological diseases and dysfunctions. A recent review presents a large body of literature demonstrating that abnormalities in epigenomes are sometimes associated with various cognitive and behavioural disorders (Franklin \& Mansuy, 2010). Among traits affected by epigenetic modifications, we find learning and memory consolidation, and pathologies such as schizophrenia, Alzheimer's disease and depression. Schaefer and co-workers observed similar effects in mice, where the histone methyltransferase complex GLP/G9a was shown (by use of conditional mutagenesis in specific neuronal regions) to affect learning, exploration and open-field behaviour (Schaefer et al., 2009). Furthermore, 
perinatal exposure to methylmercury (MeHG) is known to cause persistent changes in behaviour in mice, for example, in learning and motivational behaviour. Onischenko and colleagues showed that this is due to a long-lasting repression of the chromatin at the brain-derived neurotrophic factor $(B D N F)$ promoter region, causing a decreased hippocampal expression of the gene (Onishchenko, Karpova, Sabri, Castrn, \& Ceccatelli, 2008).

Epigenetic regulation of $B D N F$ was also found to be involved in fear extinction in mice (Bredy et al., 2007). Behavioural training produced changes in $\mathrm{H} 3$ acetylation around the p1-promoter of $B D N F$, and extinction training reversed it. When given histone deacetylase inhibitors prior to training, the mice showed enhanced long-term memory for extinction.

An interesting aspect of epigenetic variation is its sensitivity to diet and pharmacological intervention. McGowan and colleagues, in a review on this subject, show several examples of how nutrition can affect behaviour development in a variety of ways strongly indicating that the phenotypic effects are direct consequences of the induced epigenetic modifications (McGowan, Meaney, \& Szyf, 2008).

Another environmental factor shown to be associated with epigenetic modifications and subsequent behavioural effects is environmental enrichment. Supplying animal cages with substrates and furnishing to allow a wider expression of their natural behaviour is known to produce various effects on many different levels, from neuronal development to stress coping and behaviour complexity (Korte \& Olivier, 2007; van Praag, Kempermann, \& Gage, 2000). Fischer and colleagues demonstrated that enrichment could recover an impoverished learning behaviour and improve long-term memories, even when significant neuronal loss and brain atrophy had already occurred (Fischer, Sananbenesi, Wang, Dobbin, \& Tsai, 2007). This was highly correlated with chromatin modifications, specifically with histone-tail acetylation, and inhibitors of histone deacytylases were able to induce dendrite growth and synapse formation. 
Although most studies have focused on the consequences of epigenetic differences on single loci, some research indicates that large-scale variation in the entire genome may be responsible for behavioural differences. For example, Alter and co-authors studied genetically identical mice and used microarrays to examine the gene expression profiles in the hippocampus (Alter et al., 2008). They found that higher activity in an open field was associated with increased variance in the gene expression pattern. Although it is not clear how this expression variation translates to behavioural variation, the authors suggest that it may be a universal vertebrate phenomenon, perhaps linked to the degree of maturation of neurons.

\section{Transgenerational effects and inheritance of epigenetic modifications}

It should be clear from previous sections that the epigenome of an individual is shaped and modified by experiences during its lifetime. Furthermore, the epigenetic variation caused by this is an important factor behind individual differences in behaviour. The next question is whether this can transfer to coming generations, modifying the behaviour of individuals which have themselves not experienced the factors causing the epigenetic differerences.

The traditional view on this subject has been that such transgenerational transfer of acquired epigenetic variants is not possible. One reason frequently given is that the entire epigenome is believed to be erased during fertilization, to be reinstated only at a later embryonal stage. Epigenetic marks transferred across generations should thus be limited to those, which depend on genetic mechanisms. However, there is an increasing body of literature suggesting that this is not always the case. Epigenetic modifications of the genome can often be traced through several generations (Franklin \& Mansuy, 2009), and changes in DNA-methylation of several genes have been observed in sperm of both F2 and F3 males of early stressed mice (Franklin, Russig, Weiss, \& Gräff, 2010). In addition, it was recently suggested that the widely held demethylation of the genome following fertilization may in fact not exist, since in mice the observation seems to be a consequence of antigenic masking; using different methods it was 
clear that the methylation was maintained after fertilization and throughout early embryo development ( $\mathrm{Li} \&$ O'Neill, 2012). Hence, there is a clear possibility that acquired modifications in behaviour due to, for example, stressful experiences, may be transmitted to later generations, even through the germline.

In this context, researchers often make a clear distinction between germline epigenetic transmission, and other transgenerational effects (Jablonka, 2009). The reason for this is that experiences affecting an individual can indirectly also affect its offspring, without that being a true case of germline modifications. For example, stress experienced by a female may cause epigenetic changes in the developing ovas (or in the case of a male, in the sperm), and hence one might argue that any changes in the phenotypes of the offspring are direct effects rather than transgenerational. Along the same mode of reasoning, a growing embryo may have its own embryonic germ cells modified by the experiences of their mothers, so even in the $F_{2}$-generation any resulting effects could be due to direct effects. Hence, it is often argued that only effects transgressing at least to the $\mathrm{F}_{3}$-generation should be considered. Also with such strict limitations, there are many examples of true transgenerational effects (Gräff \& Mansuy, 2008; Jablonka, 2009).

One of the more dramatic examples are provided by Skinner and colleagues, who examined the long-term effects of exposure of vinclozilin, an endocrine disruptive and anti-androgen fungicide, to rats (Skinner, Anway, Savenkova, \& Gore, 2008). Exposure was experienced during the embryonic phase, but dramatic reprogramming of the brain transcriptome was detectable also in the F3-generation. The male grand-offspring of the exposed animals even displayed less anxiety-like behaviour in a light-dark box and in an elevated plusmaze, while the opposite was found for females. Excitingly, the $F_{3}$ males of the exposed line were discriminated by females from non-exposed, and were less preferred in a mate-choice test (Crews et al., 2007). This demonstrates not only transgenerational, but also possible transpopulation effects, because in the wild, it is typically males, which disperse and hence carry their epigenetic modifications to other populations. 
Along the same line of observations, Morgan and Bale exposed pregnant mice to stress during the first week of pregnancy, and then studied the F2 offspring of the males born after this embryonic exposure (Morgan \& Bale, 2011). They found that these males were dysmasculinized in morphology, physiology (reaction to stress) and behaviour (responses in tests which typically produce sexually dimorphic results). Furthermore, they observed significant differences in several micro-RNAs in brains of the dysmasculinized males, indicating that these substances play an important role in the epigenetic organisation of sexual dimorphisms in the mouse brain.

Although it is important to distinguish true germline transgenerational effects, the more short-term, direct effects may also be evolutionarily important. If the experiences acquired in one generation can affect the behaviour and phenotype of the next, this offers an alternative mode of trait transmission compared to that based on DNA-polymorphisms only. It may lead to a fast change in phenotype, which could significantly increase the fitness of the epigenotype. The previously quoted example, where intrauterine cross-fostering of mice modified the phenotype to an extent that genetic strain differences disappeared, is a good example of this (Francis et al., 2003). Such epigenetically mediated changes can easily be self-perpetuated. For example, rat offspring of mothers performing more arched-back nursing show epigenetic modifications and a different stress susceptibility, as described above (Weaver et al., 2004). In addition, female offspring of mothers performing more arched-back nursing tend to behave similarly to their own offspring, displaying more arched-back nursing and grooming than females raised by less mothers showing less of these behaviours. In this way, one realises that the transgenerational effects may remain for a long time by means of a reinstatement of the phenotype in every generation, possibly mediated by epigenetic modulation of the expression of the oxytocin receptor gene (Meaney, 2001).

Looking at transgenerational effects in this context, there are many convincing examples that this is not only common, but also that it may have substantial effects on adaptive responses. Maternal separation in young mice not only alters the DNA methylation profile in the germline of the affected males, and 
their behavioural response to aversive environments, but is also associated with a modified brain gene expression in their offspring, even when these are raised under normal conditions (Franklin et al., 2010). A corresponding transgenerational effect of early maternal separation has also been found in female mice (Weiss, Franklin, \& Vizi, 2011). My group has demonstrated transgenerational, epigenetically mediated changes in behaviour in chickens as a result of both chronic stress (Lindqvist et al., 2007) and brief events of early social isolation (Goerlich, Nätt, Elfwing, Macdonald, \& Jensen, 2012). Also "positive" experiences may transmit across generations. A nice example is provided by Arai, Li, Hartley \& Feig (2009), who showed that early exposure to environmental enrichment in mice not only enhanced long-term potentiation and associated memory formations in the affected animals, but also in their offspring.

A beautiful demonstration that evolution can act on epigenetic variation even in the absence of genetic differences is provided by the experiment by Cropley and co-authors (Cropley, Dang, Martin, \& Suter, 2012). They studied the inheritance of the pseudo-agouti phenotype in mice, which is caused by a methylated epi-allele. When they selected for this trait in an environment where the animals received methyl-donor enriched food, the frequency of the phenotype increased over the five generations included in the experiment. As stated by the authors, this shows that epigenetic variation, as well as genetic, can act as a substrate for Darwinian selection. Hence, under specified environmental conditions, adaptive epigenotypes can evolve, based on existing epigenetic variation in the population.

\section{Stress as a mediator of epigenetic effects}

It should be clear from the previous sections, that stress seems to have a profound influence on epigenetic and transgenerational effects on behaviour. Different forms of stressful challenges have been used as experimental treatments to induce such effect, for example maternal isolation and chemical exposure of various kinds (Banerjee, Arterbery, Fergus, \& Adkins-Regan, 2011; 
Champagne \& Curley, 2008; Kaminen-Ahola et al., 2010). Is this just a matter of experimental convenience, or is there something particular about the stress response, which makes it more likely than other physiological reactions to cause epigenetic changes relevant for behaviour?

Definitions of stress vary widely, according to context and author. In a recent review, Koolhaas and colleagues concluded that the concept of stress should be limited to situations where the challenges exceed the natural regulatory capacity of an animal, but conceive that such situations will always be associated with an activation of the hypothalamic-pituitary-adrenal axis and the sympathetic nervous system (Koolhaas, Bartolomucci, \& Buwalda, 2011). It is also increasingly clear that some challenges ("stressors") may actually have longlasting positive and adaptive effects on organisms and be important mediators of phenotypic plasticity, which may in turn be attributed to epigenetic variation (Costantini, Metcalfe, \& Monaghan, 2010).

Even in the absence of well-defined epigenetic mechanisms, studies have shown long-lasting and transgenerational effects of stress in various life phases. For example, Daphnia exposed to predator scents develop a protective calcium helmet, and their offspring are born with a similar protection (Agrawal, Laforsch, \& Tollrian, 1999), and offspring of crickets which had been confronted with a predator during pregnancy are more wary and prone to seek protection (Storm \& Lima, 2010). These examples provide evidence that stress may cause adaptive transgenerational effects, but do not give any information on the epigenetic effects involved (if any).

In vertebrates, it was found that raising zebra finches in experimentally enlarged broods (hence, with poorer food access) caused a decreased reproductive success in the offspring of the birds thus experiencing early stress (Naguib \& Gil, 2005; Naguib, Nemitz, \& Gil, 2006). In mammals, there is a large body of literature showing lasting, sometimes transgenerational effects of prenatal stress, of which some seem to be adaptive to some extent (Braastad, 1998; Kaiser \& Sachser, 2005). Most of the studies referred to here have not examined the possible epigenetic mechanisms involved, even though it appears 
evident that such mechanisms must play an important role. For example, although the exact mechanisms are unknown, my group has shown that stress induced modifications in hypothalamic gene expression profiles are significantly mirrored in the offspring of the affected animals (Goerlich et al., 2012; Lindqvist et al., 2007; Nätt et al., 2009).

What makes stress special in this context may be the mode of action of the steroid hormones. While peptide hormones bind to G-protein coupled receptors at the cell surface, steroids have the possibility to permeate into the cell, where they bind to their receptors, forming a protein complex which moves to the nucleus, binds to DNA and modifies gene expression (Hunter, 2012; Sapolsky, Romero, \& Munck, 2000). It is not clear how this might create long-term, and even transgenerational, epigenetic modifications, but it is at least an intriguing thought that this direct interaction exists between steroids (which are responsive to stress) and DNA (Weaver, 2009).

In connection with stress, the main steroids usually considered are the glucocorticoids cortisol or corticosterone (depending on species), which bind to the glucocorticoid receptor (GR) and then regulate various genes (Hunter, 2012). The previously discussed experiments on epigenetic effects of different mothering quality in rats have demonstrated that maternal care affects methylation of the $N r 3 c 1$ exon 1-7 (Weaver et al., 2004; 2007). This is also associated with higher levels of histone acetylation at the same promoter, indicating that stimulation of glucocorticoids can cause long-lasting epigenetic changes. Other steroids have similar modes of action, and may also respond analogously to stress. For example, sex steroids such as estrogen and testosterone are also affected, and they control a variety of sex differences in behaviour (Shepard, Michopoulos, Toufexis, \& Wilson, 2009).

Glucocorticoids have also been suggested to affect epigenetic modifications of other important behavioural genes. For example, brief immobilizations of rats caused a decrease in the expression of the brain-derived neurotrophic factor $(B D N F)$, and this was due to altered histone acetylation in promoters of the gene (Fuchikami, Morinobu, \& Kurata, 2009). The evidence 
suggested that glucocorticoids are involved in this histone modification. Similar effects have been found as a result not only of restraint stress, but also of administration of corticosterone to rats (Hunter, McCarthy, Milne, Pfaff, \& McEwen, 2009). The pathway from stress experience via glucocorticoid stimulation to epigenetic modifications of $B D N F$ and other important behavioural genes may be important for many stress-induced, long term effects, such as those resulting from early maltreatment in humans (Roth, 2012; Roth, Lubin, Funk, \& Sweatt, 2009).

\section{Evolutionary implications of transgenerational effects on behaviour}

It should be clear from this review that environmental effects can cause longterm and transgenerational epigenetic modifications which are able to change the behaviour of animals generations after the exposure. It is also clear that selection can act on epigenetic variation, effectively creating an evolutionary pathway which is independent of genetic mutations (Cropley et al., 2012). Several authors have suggested that this calls for a modified interpretation of evolutionary theory, to incorporate "soft inheritance" or "neo-lamarckism" (Jablonka, 2009; Richards, 2006). No doubt, we have moved quite some distance since the statement of Futuyma, quoted in the introduction of this review.

What is the evolutionary significance of transgenerational effects on behaviour? Strictly speaking, evolutionary development relies on three simultaneous principles: variation, heritability and selection (Futuyma, 1997). Any trait, which varies in a population, where the variation has a hereditary component, and where some variants are more reproductively efficient (selected), will evolve. Traditionally, the only source of valid variation has been considered to be (random) genetic mutations, and heritability has been regarded as the genetically mediated transfer of traits through the germline. However, with the massive new insights into transgenerational effects quoted in this review, it is difficult to see why this view should pertain.

First of all, as noted by Jablonka and others more than a decade ago, there are several different transgenerational pathways which may cause behavioural 
variation between populations and thereby offer substrate for natural selection (Jablonka \& Lamb, 1998). For example, social learning and cultural transmission of different habits across generations may cause stable between-population variation, which can offer selective advantages under the specific conditions in which they developed. As long as the social dynamics remain reasonably constant, the trait may thus evolve in a fashion similar to that seen with strictly genetically controlled traits. Furthermore, as also pointed out by Jablonka and her colleagues, behaviour variants and the ability to respond to social and cultural transmission will be related to genotype. Some genotypes may be more prone to such transmission than others, so there is a strong interaction between culture and genes.

One might therefore imagine that also epigenetic variation could affect cultural transmission ability. Although the evidence for this is not compelling so far (because few people have looked for this), this would add a layer of complexity to the theory of evolution. This review has demonstrated how genes and environment interact to create epigenetic variation, and it is an intriguing thought that such epigenetic variation in turn could affect the efficiency of the transgenerational transmission process.

One could envisage a situation where cultural transmission is affected by epigenetic variation and vice versa - would it be possible that generations of such cultural transmission of a particular epigenetic variant could cause a stabilization of this epigenetic variant? The rationale would be that if a particular epiallele confers selective advantage, then genetic mutations facilitating this epiallele would have a similar advantage. In this way, over generations, a pure or facilitatated epigenetic mark may develop into an obligatory mark, sensu Richards (Richards, 2006). In our own research, we found that domesticated chickens are hypermethylated in more than $70 \%$ of those loci which are differentially methylated compared to their ancestors, the Red Junglefowl (Nätt et al., 2012). This indicates that chickens have acquired many novel methylations during domestication, and we also found that several of them are stable over many generations. Although this can be explained by different genetic 
mechanisms, it can not be excluded that many of the methylation differences are the result of a gradual evolution from pure to obligatory epigenetic marks.

Yet another speculative possibility, which should receive attention in future research is the possibility that epigenetic modifications predispose for genetic mutations. There is no actual evidence to support such a suggestion today, but it remains a plausible possibility that a gene, which becomes silenced through epigenetic modifications may be less attended to by DNA-repair mechanisms, and hence be more vulnerable to mutation. This could be another route of action through which the environment could potentially affect DNA in an adaptive manner.

At the present state, the possible adaptational value of transgenerational effects remains hypothetical. There is no question that some examples support such a conclusion. As discussed above, crickets are able to modify the antipredator response of their offspring in a seemingly adaptive manner in response to their own experienced predation risk (Storm \& Lima, 2010). Our group found that a modified foraging behaviour in chickens, in response to an undpredictable day length, was mirrored in the female offspring; also the associated change in brain gene expression profile was similar in the offspring (Nätt et al., 2009). And, as shown by the earlier discussed experiment by Cropley and co-workers, selection for epigenetic variation can cause an increase in epialleles over several generations, much in accordance with what we know from selection on genetic mutations (Cropley et al., 2012).

Strong arguments against the idea of extending evolutionary theory to incorporate soft inheritance and epigenetics has recently been formulated (Dickins \& Rahman, 2012). These authors argue that extending epigenetic findings to evolutionary theory confounds proximate and ultimate levels of explanation, partly because much epigenetic variation is under strict genetic control. Although critical to some of the wide applications of epigenetics, these authors of course do not deny the existence of transgenerational effects as such, but claim that this can be perfectly well incorporated into the general and prevailing modern synthesis in evolutionary biology. 


\section{Conclusions}

In this review, I have collected papers mainly from the last ten or so years, which provide overwhelming evidence that behavioural changes caused by various environmental challenges can be transmitted to offspring, sometimes persisting in several generations. This is sometimes due to epigenetic effects, which modify gene expression patterns and are (at least in some cases) inherited through the germline. Even though there has been a focus on studying pathological or other maladaptive behavioural changes resulting from stress of various kinds, some evidence does exist that the transgenerational effects may sometimes be adaptive and increase the fitness of the individuals in the affected family.

This research is still in its infancy, and although the correlational evidence is often compelling, there is still a lack of understanding of the underlying mechanisms, as well as the functional and evolutionary consequences of transgenerational effects. With a few exceptions (for example the studies by the Meaney and Champagne labs), little is known about how a particular epigenetic modification of a certain locus translates into a particular behaviour phenotype. Most studies so far are based on correlational evidence, so proper experimental demonstrations of cause and effect relationships between epigenetic modifications and behaviour are largely lacking. And although circumstantial evidence suggest that some epigenetically mediated behaviour modifications may confer fitness advantages, this remains to be properly demonstrated in longterm studies combining evolutionary theory and molecular biology.

Although much work is still needed, it is fair to say that the days are gone when biologists could safely argue that acquired behavioural traits can not be transmitted to the next generation. I would suggest that this is a compelling example of a shift in paradigm in behavioural and evolutionary biology.

\section{Acknowledgements}


I am grateful for the input from dr Dominic Wright. The work on epigenetics and behaviour in my group is supported by the Swedish Research Council (VR) and the Swedish Research Council for Environment, Agricultural Sciences and Spatial Planning (Formas). It is conducted within the framework of the $\mathrm{LiU}$ Neuroscience initiative, and the Swedish Centre of Excellence in Animal Welfare Research. 


\section{References}

Agrawal, A. A., Laforsch, C., \& Tollrian, R. (1999). Transgenerational induction of defences in animals and plants. Nature, 401(6748), 60-63.

Alter, M. D., Rubin, D. B., Ramsey, K., Halpern, R., Stephan, D. A., Abbott, L. F., \& Hen, R. (2008). Variation in the large-scale organization of gene expression levels in the hippocampus relates to stable epigenetic variability in behavior. PLoS ONE, 3(10), e3344.

Arai, J. A., Li, S., Hartley, D.M., \& Feig, L.A. (2009). Transgenerational rescue of a genetic defect in long-term potentiation and memory formation by juvenile enrichment. Journal of Neuroscience, 29 (5), 1496-1502.

Banerjee, S. B., Arterbery, A. S., Fergus, D. J., \& Adkins-Regan, E. (2012).

Deprivation of maternal care has long-lasting consequences for the hypothalamic-pituitary-adrenal axis of zebra finches. Proceedings of the Royal Society B: Biological Sciences, 279, 759-766

Barnard, C. J. (2004). Animal Behaviour. Prentice Hall.

Braastad, B. (1998). Effects of prenatal stress on behaviour of offspring of laboratory and farmed mammals. Applied Animal Behaviour Science, 61, 159180

Bredy, T. W., Wu, H., Crego, C., Zellhoefer, J., Sun, Y. E., \& Barad, M. (2007). Histone modifications around individual BDNF gene promoters in prefrontal cortex are associated with extinction of conditioned fear. Learning \& memory, 14(4), 268-276.

Champagne, F. A., \& Curley, J. P. (2008). Maternal regulation of estrogen receptor alpha methylation. Current Opinion in Pharmacology, 8(6), 735-739.

Champagne, F. A., Weaver, I. C. G., Diorio, J., Dymov, S., Szyf, M., \& Meaney, M. J. (2006). Maternal care associated with methylation of the estrogen receptoralpha1b promoter and estrogen receptor-alpha expression in the medial preoptic area of female offspring. Endocrinology, 147(6), 2909-2915.

Costantini, D., Metcalfe, N. B., \& Monaghan, P. (2010). Ecological processes in a hormetic framework. Ecology Letters, 13(11), 1435-1447.

Crews, D., Gore, A. C., Hsu, T. S., Dangleben, N. L., Spinetta, M., Schallert, T., Anway, M. D., et al. (2007). Transgenerational epigenetic imprints on mate 
preference. Proceedings of the National Academy of Sciences, 104(14), 59425946.

Cropley, J. E., Dang, T. H. Y., Martin, D. I. K., \& Suter, C. M. (2012). The penetrance of an epigenetic trait in mice is progressively yet reversibly increased by selection and environment. Proceedings of the Royal Society B: Biological Sciences, 279(1737), 2347-2353.

Curley, J. P., Jensen, C. L., Mashoodh, R., \& Champagne, F. A. (2011). Social influences on neurobiology and behavior: Epigenetic effects during development. Psychoneuroendocrinology, 36(3), 352-371.

Dickins, T. E., \& Rahman, Q. (2012). The extended evolutionary synthesis and the role of soft inheritance in evolution. Proceedings of the Royal Society B: Biological Sciences, 279(1740), 2913-2921.

Fischer, A., Sananbenesi, F., Wang, X., Dobbin, M., \& Tsai, L.-H. (2007). Recovery of learning and memory is associated with chromatin remodelling. Nature, 447(7141), 178-182.

Francis, D. D., Szegda, K., \& Campbell, G. (2003). Epigenetic sources of behavioral differences in mice. Nature Neuroscience, 6(5), 445-446.

Franklin, T. B., \& Mansuy, I. M. (2009). Epigenetic inheritance in mammals: Evidence for the impact of adverse environmental effects. Neurobiology of Disease, 1-5.

Franklin, T. B., \& Mansuy, I. M. (2010). The prevalence of epigenetic mechanisms in the regulation of cognitive functions and behaviour. Current Opinion in Neurobiology, 1-9.

Franklin, T., Russig, H., Weiss, I., \& Gräff, J. (2010). Epigenetic transmission of the impact of early stress across generations. Biological Phychiatry, 68, 408-415.

Fuchikami, M., Morinobu, S., \& Kurata, A. (2009). Single immobilization stress differentially alters the expression profile of transcripts of the brain-derived neurotrophic factor (BDNF) gene and histone acetylation at its promoters in the rat hippocampus. The International Journal of Neuropsychopharmacology, $12,73-82$.

Futuyma, D. J., Futuyma. (1997). Evolutionary Biology (3rd ed.). Sinauer Associates.

Gertz, J., Varley, K. E., Reddy, T. E., Bowling, K. M., Pauli, F., Parker, S. L., Kucera, K. 
S., et al. (2011). Analysis of DNA Methylation in a Three-Generation Family Reveals Widespread Genetic Influence on Epigenetic Regulation. (W. A. Bickmore, Ed.)PLoS Genetics, 7(8), e1002228.

Goerlich, V. C., Nätt, D., Elfwing, M., Macdonald, B., \& Jensen, P. (2012).

Transgenerational effects of early experience on behavioral, hormonal and gene expression responses to acute stress in the precocial chicken. Hormones and Behavior, 61, 711-718.

Gräff, J., \& Mansuy, I. M. (2008). Epigenetic codes in cognition and behaviour. Behavioural Brain Research, 192(1), 70-87.

Hofmann, H. A. (2003). Functional genomics of neural and behavioral plasticity. Journal of Neurobiology, 54(1), 272-282.

Hunter, R. G. (2012). Epigenetic effects of stress and corticosteroids in the brain, Frontiers in cellular neuroscience, 6: 1-8. doi:10.3389/fncel.2012.00018

Hunter, R. G., McCarthy, K. J., Milne, T. A., Pfaff, D. W., \& McEwen, B. S. (2009). Regulation of hippocampal H3 histone methylation by acute and chronic stress. Proceedings of the National Academy of Sciences, 106(49), 2091220917.

Jablonka, E. (2009). Transgenerational epigenetic inheritance: prevalence, mechanisms, and implications for the study of heredity and evolution. Quarterly Review of Biology, 84(2), 131-176.

Jablonka, E., Lamb, M. \& Avital, E. (1998). “Lamarckian” mechanisms in darwinian evolution. Trends in Ecology \& Evolution, 13, 206-210.

Jensen, P. (2006). Domestication-From behaviour to genes and back again ţ. Applied Animal Behaviour Science, 97(1), 3-15.

Jöngren, M., Westander, J., Nätt, D., \& Jensen, P. (2010). Brain gene expression in relation to fearfulness in female red junglefowl (Gallus gallus). Genes, Brain and Behavior, 9(7), 751-758.

Kaiser, S., \& Sachser, N. (2005). The effects of prenatal social stress on behaviour: mechanisms and function. Neuroscience \& Biobehavioral Reviews, 29(2), 283294.

Kaminen-Ahola, N., Ahola, A., Maga, M., Mallitt, K.-A., Fahey, P., Cox, T. C., Whitelaw, E., et al. (2010). Maternal Ethanol Consumption Alters the Epigenotype and the Phenotype of Offspring in a Mouse Model. PLoS Genetics, 
6(1), e1000811.

Kappeler, L. (2010). Epigenetics and parental effects. BioEssays, 32(9), 818-827.

Koolhaas, J., Bartolomucci, A., \& Buwalda, B. (2011). Stress revisited: A critical evaluation of the stress concept. Neuroscience \& Biobehavioral Reviews, 35(5), 1291-1301.

Korte, S., \& Olivier, B. (2007). A new animal welfare concept based on allostasis. Physiology \& Behavior, 92(3), 422.428.

Lester, B. M., Tronick, E., Nestler, E., Abel, T., Kosofsky, B., Kuzawa, C. W., Marsit, C. J., et al. (2011). Behavioral epigenetics. Annals of the New York Academy of Sciences, 1226(1), 14-33.

Li, Y., \& O'Neill, C. (2012). Persistence of Cytosine Methylation of DNA following Fertilisation in the Mouse. PLoS ONE, 7(1), e30687.

Lindqvist, C., Janczak, A. M., Nätt, D., Baranowska, I., Lindqvist, N., Wichman, A., Lundeberg, J., et al. (2007). Transmission of Stress-Induced Learning Impairment and Associated Brain Gene Expression from Parents to Offspring in Chickens. (C. Allen, Ed.)PLoS ONE, 2(4), e364.

McGowan, P. O., Meaney, M. J., \& Szyf, M. (2008). Diet and the epigenetic (re)programming of phenotypic differences in behavior. Brain Research, $1237,12-24$.

McGowan, P. O., Sasaki, A., D'Alessio, A. C., Dymov, S., Labonté, B., Szyf, M., Turecki, G., et al. (2009). Epigenetic regulation of the glucocorticoid receptor in human brain associates with childhood abuse. Nature Neuroscience, 12(3), 342-348.

Meaney, M. (2001). Maternal care, gene expression, and the transmission of individual differences in stress reactivity across generations. Annual Review of Neuroscience, 24, 1161-1192.

Morgan, C. P., \& Bale, T. L. (2011). Early Prenatal Stress Epigenetically Programs Dysmasculinization in Second-Generation Offspring via the Paternal Lineage. Journal of Neuroscience, 31(33), 11748-11755.

Naguib, M., \& Gil, D. (2005). Transgenerational body size effects caused by early developmental stress in zebra finches. Biology Letters, 1, 95-97.

Naguib, M., Nemitz, A., \& Gil, D. (2006). Maternal developmental stress reduces reproductive success of female offspring in zebra finches. Proceedings of the 
Royal Society B: Biological Sciences, 273(1596), 1901-1905.

Nätt, D., Lindqvist, N., Stranneheim, H., Lundeberg, J., Torjesen, P. A., \& Jensen, P. (2009). Inheritance of Acquired Behaviour Adaptations and Brain Gene Expression in Chickens. PLoS ONE, 4(7), e6405.

Nätt, D., Rubin, C., Wright, D., \& Johnsson, M. (2012). Heritable genome-wide variation of gene expression and promoter methylation between wild and domesticated chickens. BMC Genomics, 13: 59

Oberlander, T. F., Weinberg, J., Papsdorf, M., Grunau, R., Misri, S., \& Devlin, A. M. (2008). Prenatal exposure to maternal depression, neonatal methylation of human glucocorticoid receptor gene (NR3C1) and infant cortisol stress responses. Epigenetics, 3(2), 97-106.

Onishchenko, N., Karpova, N., Sabri, F., Castrn, E., \& Ceccatelli, S. (2008). Longlasting depression-like behavior and epigenetic changes of BDNF gene expression induced by perinatal exposure to methylmercury. Journal of neurochemistry, 106(3), 1378-1387.

Pearce, J. M. (2008). Animal Learning and Cognition, 3rd Edition: An Introduction (3rd ed.). Psychology Press.

Richards, E. (2006). Inherited epigenetic variation-revisiting soft inheritance. Nature Reviews Genetics, 7, 395-401.

Roth, T. L. (2012). Epigenetics of neurobiology and behavior during development and adulthood. (J. Zevin \& K. Bath, Eds.)Developmental Psychobiology, 54(6), 590-597.

Roth, T. L., Lubin, F. D., Funk, A. J., \& Sweatt, J. D. (2009). Lasting epigenetic influence of early-life adversity on the BDNF gene. Biological psychiatry, 65(9), 760-769.

Sapolsky, R. M., Romero, L. M., \& Munck, A. U. (2000). How do glucocorticoids influence stress responses? Integrating permissive, suppressive, stimulatory, and preparative actions. Endocrine reviews, 21(1), 55-89.

Schaefer, A., Sampath, S. C., Intrator, A., Min, A., Gertler, T. S., Surmeier, D. J., Tarakhovsky, A., et al. (2009). Control of cognition and adaptive behavior by the GLP/G9a epigenetic suppressor complex. Neuron, 64(5), 678-691.

Shepard, K. N., Michopoulos, V., Toufexis, D. J., \& Wilson, M. E. (2009). Genetic, epigenetic and environmental impact on sex differences in social behavior. 
Physiology \& Behavior, 97(2), 157-170. d

Skinner, M., Anway, M., Savenkova, M., \& Gore, A. (2008). Transgenerational epigenetic programming of the brain transcriptome and anxiety behavior. PLoS ONE, 3 (11), e3745.

Storm, J. J., \& Lima, S. L. (2010). Mothers Forewarn Offspring about Predators: A Transgenerational Maternal Effect on Behavior. The American Naturalist, 175(3), 382-390.

van Praag, H., Kempermann, G., \& Gage, F. H. (2000). Neural consequences of environmental enrichment. Nature Reviews Neuroscience, 1, 191-198.

Weaver, I. (2009). Epigenetic effects of glucocorticoids. Seminars in Fetal and Neonatal Medicine, 14, 143-150.

Weaver, I. C. G., Cervoni, N., Champagne, F. A., D'Alessio, A. C., Sharma, S., Seckl, J. R., Dymov, S., et al. (2004). Epigenetic programming by maternal behavior. Nature Neuroscience, 7(8), 847-854.

Weaver, I. C. G., D'Alessio, A. C., Brown, S. E., Hellstrom, I. C., Dymov, S., Sharma, S., Szyf, M., et al. (2007). The transcription factor nerve growth factor-inducible protein a mediates epigenetic programming: altering epigenetic marks by immediate-early genes. Journal of Neuroscience, 27(7), 1756-1768.

Weaver, I. C. G., Meaney, M. J., \& Szyf, M. (2006). Maternal care effects on the hippocampal transcriptome and anxiety-mediated behaviors in the offspring that are reversible in adulthood. Proceedings of the National Academy of Sciences of the United States of America, 103(9), 3480-3485.

Weiss, I., Franklin, T., \& Vizi, S. (2011). Inheritable effect of unpredictable maternal separation on behavioral responses in mice. Frontiers in Behavioral Neuroscience, 5, doi: 10.3389/fnbeh.2011.00003 


\section{Legend to figure}

\section{Fig 1:}

Some of the possible pathways through which the epigenome interacts with environment and genes and exerts effects on behaviour. Each of them relies on specific mechanisms, which are discussed in the text. 


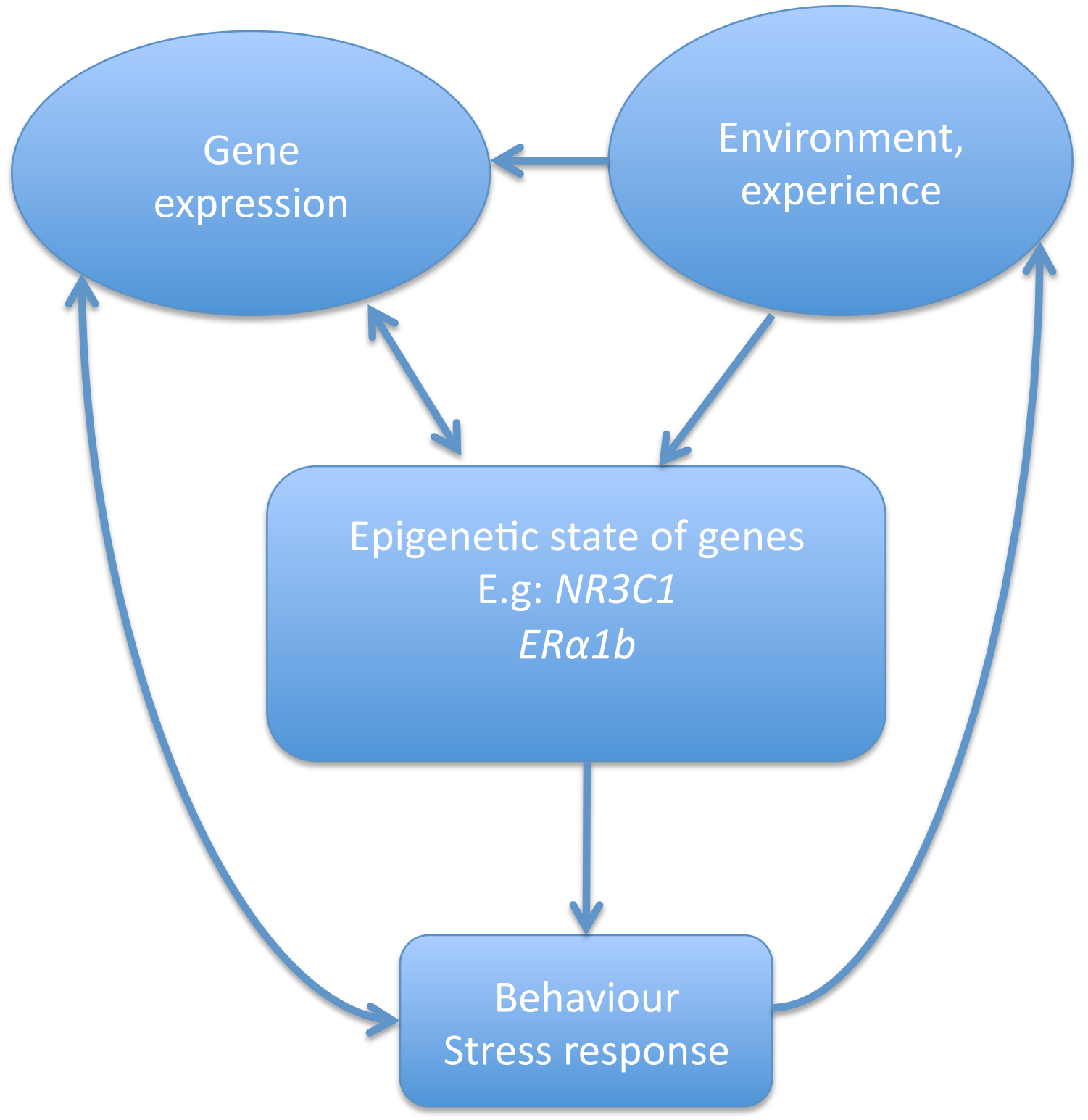

